(C) 1998 Cambridge University Press

\title{
Find The Errors, Get The Better: Enhancing Machine Translation via Word Confidence Estimation
}

\author{
Ngoc-Quang Luong, Laurent Besacier, Benjamin Lecouteux \\ Laboratoire d'Informatique de Grenoble, Campus de Grenoble \\ 41, Rue des Mathématiques, BP53, F-38041 Grenoble Cedex 9, France
}

( Received $M M / D D / Y Y Y Y$; revised $M M / D D / Y Y Y Y$ )

\begin{abstract}
This article presents two novel ideas of improving the Machine Translation (MT) quality by applying the word-level quality prediction for the second pass of decoding. In this manner, the word scores estimated by Word Confidence Estimation (WCE) systems help to reconsider the MT hypotheses for selecting a better candidate rather than accepting the current sub-optimal one. In the first attempt, the selection scope is limited to the MT N-best list, in which our proposed re-ranking features are combined with those of the decoder for re-scoring. Then, the search space is enlarged over the entire search graph, storing many more hypotheses generated during the first pass of decoding. Over all paths containing words of the N-best list, we propose an algorithm to strengthen or weaken them depending on the estimated word quality. In both methods, the highest-score candidate after the search becomes the official translation. The results obtained show that both approaches advance the MT quality over the one-pass baseline, and the Search Graph Re-decoding achieves more gains (in BLEU score) than N-best List Re-ranking method.
\end{abstract}

\section{Introduction}

The core idea of Statistical Machine Translation (SMT) is to generate all possible hypotheses for a given input sentence, then search for the hypothesis of highest score to become the output. The score used to judge the candidates consists of various factors, e.g. language model, translation model, reordering model, etc. Since the state-of-the-art MT models are imperfect, their outputs might not meet the user's expectations. More specifically, the MT output perhaps beats the others under the decoder's assessment, yet in many cases remains sub-optimal according to readers' viewpoint. For instance, by looking at the N-best list presented in Table 1 , it is not hard to realize that the third ranked hypothesis is more valuable than the current 1-best, with only one "Shift" operation ("association udf" $\rightarrow$ "udf association") is required to become the reference (post-edition), although its model score is lower. Therefore, improving MT performance by adding more objective and decoder-independent features is a roadmap that many researchers are attempting. 


\begin{tabular}{ll}
\hline Source (fr) & $\begin{array}{l}\text { l'association udf hausse le ton et somme le nouveau centre } \\
\text { de ne plus utiliser son sigle. }\end{array}$ \\
Post-edition (en) & $\begin{array}{l}\text { the udf association increases the tone and commands the } \\
\text { new centre to stop using its acronym. }\end{array}$ \\
\hline Hypothesis $e^{1}$ & the association udf increase the tone and after the new \\
centre to stop using its acronym. & the association udf rise the tone and warn the new \\
Hypothesis $e^{2}$ & $\begin{array}{l}\text { centre to stop using the acronym. } \\
\text { the association udf increases the tone and commands the } \\
\text { new centre to stop using its acronym. } \\
\text { the association udf tone and increase the amount } \\
\text { the new centre to use its acronym. } \\
\text { the association udf increase the tone and warn the new } \\
\text { centre not to use its abbreviation. }\end{array}$ \\
\hline
\end{tabular}

Table 1. With state-of-the-art SMT systems, the 1-best is not always optimal

Joining these endeavors, this article contributes two novel ideas for generating a better candidate from the quality labels predicted for words of the current MT output. More specifically, we automatically identify the good and bad words (word confidence estimation), then exploit them as additional indicators to "decode" one more time for acquiring more valuable translation. There are two spaces over which the search can be conducted:

- The MT $\boldsymbol{N}$-best list: after decoding, besides the official translation $e^{1}$, the decoder generates also $N-1$ other alternative hypotheses $\left\{e^{2}, e^{3}, \ldots, e^{N}\right\}$ with lower scores. The totality of these $N$ hypotheses is known as the $N$-best list. Working on this list, we integrate into the current objective scoring function with our proposed parameters based on WCE scores, then re-rank it by this enriched function.

- The MT Search Graph (SG) can be considered as a "vast warehouse" storing all possible hypotheses generated by the SMT decoder. Our idea when exploring this huge space is that the overall scores of all paths containing tagged (error/ no error) words will be modified due to their quality predicted beforehand by WCE. More precisely, the score is strengthened (increased) in case of the path contains good words and weakened (decreased) otherwise.

Word Confidence Estimation (WCE) is not a novel topic, although it is still understudied compared to research on Sentence-level Quality Estimation. Nevertheless, the idea of using it to enhance MT quality has not been widely investigated in literature. To the best of our knowledge, if we ignore our previously published conference papers, the confidence score (called Goodness) is applied only once in the work of Nguyen, Huang and Al-Onaizan (2011) to re-rank the $N$-best list, in a different way from our approach.

The rest of this article is organized as follows. After reviewing some related work in Section 2, we investigate the correlation between our proposed scores (built using WCE) and other MT quality metrics (BLEU, TER, TERp-A) in Section 3. Section 
4 presents the WCE system building. Sections 5 details the idea of using WCE to re-rank the $N$-best list. Another way of exploiting it to re-decode the SMT search graph is discussed in Section 6. Section 7 compares the two methods, concludes the article and points some perspectives.

\section{Related Work 2.1 Word Confidence Estimation}

Confidence Estimation (CE) is the task of identifying the correct parts and detecting the translation errors in MT output. If the quality is predicted for each word, this becomes WCE. The interesting uses of WCE include: pointing out the words that need to be corrected by the post-editor, telling readers about the reliability of a specific portion, and selecting the best segments among options from multiple translation systems for combination.

To deal with this problem, various approaches have been proposed. They mainly focus on two principal issues: the features to represent each word and the Machine Learning (ML) methods to train the classifier. In this domain's pioneer work, Blatz, Fitzgerald, Foster, Gandrabur, Goutte, Kulesza, Sanchis and Ueffing (2003) combine several features using neural network and Naïve Bayes learning algorithms. One of the most effective feature combinations is the Word Posterior Probability (WPP) as suggested by Ueffing, Macherey and Ney (2003) associated with IBM-model based features (Blatz, Fitzgerald, Foster, Gandrabur, Goutte, Kulesza, Sanchis and Ueffing (2004)). Basically, WPP is the likelihood of the word occurring in the target sentence, given the source sentence. Numerous knowledge sources have been proposed to calculate it, such as word graphs, N-best lists, etc. To quantify it, the key point is to determine sentences in N-best lists that contain the word $e$ under consideration in a fixed position $i$.

Ueffing and Ney (2005) propose an approach for phrase-based translation models: a phrase is a sequence of contiguous words and is extracted from the word-aligned bilingual training corpus. The confidence value of each word is then computed by summing over all phrase pairs in which the target part contains this word. Xiong, Zhang and Li (2010) incorporate linguistic features (the word itself, part-of-speech (POS) and null-link feature) with WPP and train their Maximum Entropy classifier, allowing considerable gains in comparison to the one using only WPP features. The novel features from source side, alignment context, and dependency structure (Nguyen et al. 2011) advance the error prediction accuracy of the baseline system using WPP and POS features in both F-score and Pearson correlation with human judgment. Other approaches are based on external features, which are independent from MT system's resources (Soricut and Echihabi (2010), Felice and Specia (2012)), allowing to cope with various MT systems, such as statistical, rule-based, etc.

Recent workshops on MT (WMT 2013, 2014 and 2015) launched WCE as an evaluation shared task. In WMT 2013, while Han, Lu, Wong, Chao, He, and Xing (2013); Luong, Lecouteux and Besacier (2013) employ the Conditional Random Fields (CRF) model (Lafferty, McCallum and Pereira (2001)) to build classifiers, 
Bicici (2013) presents the global learning model by dynamic training with adaptive weight updates in the perceptron training algorithm. Concerning features: (Bicici, 2013) presents the "common cover links" (the links that point from the leaf node containing this word to other leaf nodes in the same subtree of the syntactic tree). (Han et al. 2013) focus on various n-gram combinations of target words. (Luong et al. 2013) integrated a number of new indicators relying on graph topology, pseudo reference, syntactic behavior (constituent label, distance to the semantic tree root) and polysemy characteristic.

In WMT 2014, Wisniewski, Pécheux, Allauzen and Yvon (2014) exploit random forest classifier and build only 16 dense and continuous features of two categories: association features between source sentence and each target word, and fluency features describing the quality of the translation hypotheses. Meanwhile, confusion network, word lexicon and POS tags are the main resources to form the feature set of the systems of Camargo-de-Souza, González-Rubio, Buck, Turchi and Negri (2014).

In WMT 2015, deep neural network is employed by Kreutzer, Schamoni, and Riezler (2015) to learn continuous feature representations from bilingual contexts. Their network is firstly trained by initializing the word lookup-table with distributed word representation, and then adapted to the WCE task via a backpropagation process, which aims to minimize the word prediction errors using stochastic gradient descent. Meanwhile, the conventional sequence labeling technique, CRF, is applied by many other participants (Shah, Logacheva, Paetzold, Blain, Beck, Bougares and Specia (2015), Logacheva, Hokamp, and Specia (2015), Shang, Cai, and Ji (2015)) to train their classifiers. Beside using baseline features, Tezcan, Hoste, Desmet, and Macken (2015) propose new ones to capture the accuracy (meaning transfer from source to target sentence) using word and phrase alignments, and the fluency (target sentence wellformedness level) via training language models on word surface forms and on part-of-speech tags.

As stated above, the main goal of this article is to apply WCE outputs in the second pass of decoding (i.e. for re-ranking the SMT $N$-best list, as well as redecoding the Search Graph). Therefore, it is interesting to investigate how SMT multiple-pass decoding was examined in the literature in the next section.

\subsection{SMT Multiple-Pass Decoding}

Among related work concerning this issue, we observe some prominent ideas. The first direction focuses on proposing additional Language Models. Kirchhoff and Yang (2005) train one word-based 4-gram model (with modified Kneser-Ney smoothing) and one factored trigram model, then combine them with seven decoder scores for re-ranking $N$-best lists of several SMT systems. Their proposed LMs increase the translation quality of the baselines (measured by BLEU score) from 21.6 to 22.0 (Finnish - English), or from 30.5 to 31.0 (Spanish - English). Meanwhile, Zhang, Almut, and Stephan (2006) experiment with a distributed LM where each server, among the total of 150, hosts a portion of the data and responses its client, allowing them to exploit an extremely large corpus (2.7 billion word English Gi- 
gaword) for estimating N-gram probability. The quality of their Chinese - English hypotheses after the re-scoring process by using this LM is improved by $4.8 \%$ (from BLEU 31.44 to 32.64 , oracle BLEU score $=37.48$ ).

In another direction, several authors propose to replace the current decoder's linear scoring function by more efficient functions. Ueffing and Ney (2007) linearly combine sentence-level WPP with scores assigned by the underlying SMT system and additional language model scores . Sokolov, Wisniewski and Yvon (2012) learn their non-linear scoring function in a learning-to-rank paradigm, applying Boosting algorithm. Their gains on the WMT' $\{10,11,12\}$ are modest yet consistent and higher than those based on linear scoring functions. Duh and Kirchhoff (2008) use Minimum Error Rate Training (MERT) (Och (2003)) as a weak learner and build their own solution, BoostedMERT, a highly-expressive re-ranker created by voting among multiple MERT ones. Their proposed model considerably beats the decoder's log-linear model (43.7 vs. 42.0 BLEU) in IWSLT 2007 Arabic - English task. Aiming at lattice rescoring methods for large-scale SMT, Blackwood (2010) proposes a linearized lattice minimum Bayes-risk decoding method based on efficient path counting transducers. He also suggests to combine multiple SMT lattices, allowing the decoder to operate on a much richer and more diverse searching space, thus improves the translation quality over several language pairs. Applying solely goodness (the sentence confidence) scores, (Nguyen et al. 2011) obtain slight yet consistent TER reductions ( 0.007 and 0.006 on the dev and test set) after a 5 -list re-ranking for their SMT hypotheses. This latter work is the most related to our paper, yet discrepant in some points: (1) our proposed sentence scores are computed based on word confidence labels; (2) we perform a study of the use of WCE for Nbest reranking and assess its usefulness in a simulated interactive scenario (using oracle WCE labels); and (3) we also use, in the second part of this paper, the WCE score to re-decode the full SMT search graph.

\section{Word Quality Scores and MT Quality Metrics}

Before exploiting WCE scores to improve MT quality, we first investigate the correlation between sentence-level scores (obtained from WCE labels) and conventional evaluation scores, including BLEU (Papineni, Roukos, Ard and Zhu, (2002)), TER and TERp-A (Snover (2008)). For each output sentence, a word quality score (WQS) is defined by the ratio of the number of words predicted as "Good" by WCE system over its total number of words:

$$
W Q S=\frac{\#^{\prime \prime} G^{\prime \prime}(\text { good }) \text { words }}{\# \text { words }}
$$

In other words, we are trying to examine whether the high percentage of " $G$ " (good) words (predicted by WCE system) in a MT output ensure its possibility of having a better BLEU and low TER (TERp-A) value. This investigation is a strong

prerequisite for further experiments in order to verify that WCE scores do not bring additional "noise" to the candidates' re-judgement process. In this investigation, we compute WQS over a French - English dataset (encompassing total of 10,881 
1-best translations), taken from news corpora of the WMT evaluation campaign (from 2006 to 2010). The post-editions of these translations are generated by using a crowdsourcing platform: Amazon Mechanical Turk. Matching MT hypotheses against post-editions using TERp- $\mathrm{A}^{1}$, a tuned version of $\mathrm{TERp}^{2}$, enables us not only to compute TER and TERp-A scores, but also to determine the word quality labels ("G", "B"), and then WQS. More details of the corpus can be found in (Potet, Rodier, Besacier and Blanchon (2012)) and those of the quality label determination with TERp-A are depicted in (Luong 2012). Once TERp-A labels are obtained, we convert them into binary labels "G", "B" (see Section 4.2 for conversion details). Once we have three conventional scores (BLEU, TER, TERp-A) for each sentence, we plot each against WQS. Here, we use the "oracle" labels in order to verify the correlation with conventional evaluation metrics, but obviously, the predicted labels will be used for the two-pass SMT described in the next section. BLEU, TER and TERp-A scores are calculated after matching with post-editions. The results are plotted in Figure 1, where the $y$ axis shows the " $G$ " (good) word percentage, and the $x$ axis shows BLEU (1a), TER (1b) or TERp-A (1c) scores. It can be seen from

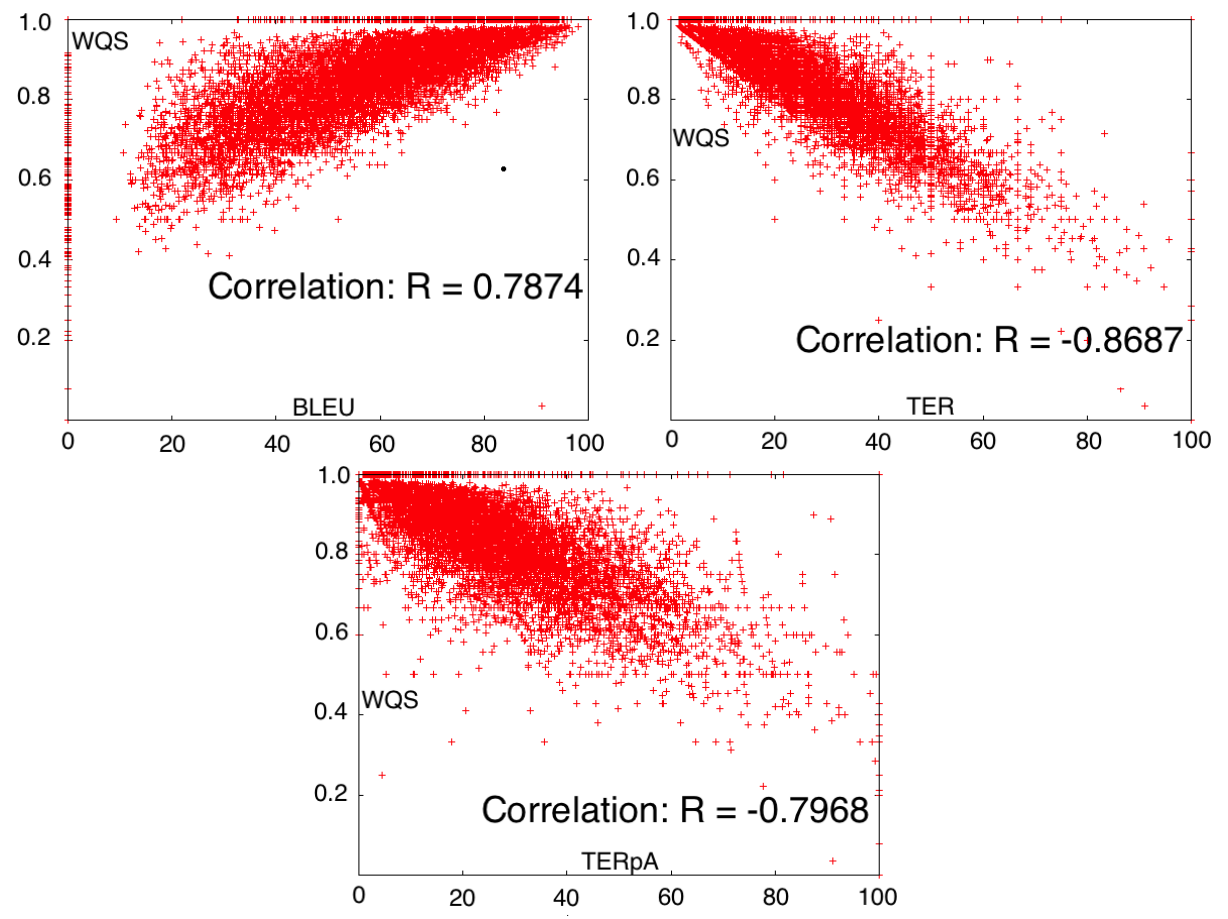

Fig. 1. The correlation between WQS in a sentence and its overall quality measured by:

(a) BLEU, (b) TER and (c) TERp-A metrics

\footnotetext{
${ }^{1}$ https://github.com/snover/terp

2 TERp is an extension of TER (Translation Edit Rate) that uses phrasal substitutions (using automatically generated paraphrases), stemming, synonyms, relaxed shifting constraints and other improvements.
} 
Figure 1 that the majority of points (the densest areas) in all three cases conform the common tendency. In Figure 1a, the higher "G" percentage, the higher BLEU score is. Conversely, in Figure 1b (Figure 1c), the higher "G" percentage, the lower TER (TERp-A) is. Furthermore, these high correlations are quantified by the high positive (0.7814 for BLEU) and negative (-0.8687 for TER, and -0.7968 for TERpA) correlation scores. We notice some outliers, i.e. sentences with most or almost all words labeled "good", yet still have low BLEU or high TER (TERp-A) scores. This phenomenon is to be expected when many unknown source words are not translated or when the unique reference is simply too far from the hypothesis. Nevertheless, the information extracted from oracle WCE labels seems useful to assess the MT hypotheses in the second pass.

\section{Experimental Setting}

\subsection{Dataset, N-best List and Search Graph Preparation}

From a dataset of 10,881 French sentences, we applied a Moses-based SMT system to generate their English hypotheses. In our SMT system, the translation model is trained on the Europarl and News parallel corpora of ${\mathrm{WMT} 10^{3}}^{(1,638,440 \text { sen- }}$ tences), and the target language model is trained by the SRILM toolkit (Stolcke 2002) on a news monolingual corpus of WMT10 (48,653,884 sentences). Next, human translators were invited to correct MT outputs, giving us the post editions. More details on this post-edited corpus can be found in (Potet et al. 2012). The set of triples (source, hypothesis, post edition) was then divided into the training set (10000 first triples) and test set (881 remaining ones). The WCE classifier was trained over all 1-best hypotheses of the training set.

The $N$-best list $(N=1000)$ with associated alignment information is also obtained on the test set $(1000 * 881=881000$ sentences $)$ by using options "- $n$-bestlist" and "-print-alignment-info-in-n-best" of Moses (version 2009-04-13) (Koehn, Hoang, Birch, Callison-Burch, Federico, Bertoldi, Cowan, Shen, Moran, Zens, Dyer, Bojar, Constantin, and Herbst (2007)). Besides, the SGs are extracted by some parameter settings: "-output-search-graph", "-search-algorithm 1" (using cube pruning) and "-cube-pruning-pop-limit 5000" (adds 5000 hypotheses to each stack). We then store the SG for each source sentence in a separated file, and the average size is $43.8 \mathrm{MB}$.

\subsection{WCE System Building}

We employ the Conditional Random Fields (Lafferty et al. 2001) (CRFs) as our machine learning method, with WAPITI toolkit ${ }^{4}$ (Lavergne, Cappé and Yvon (2010)), to train the WCE model. A number of knowledge resources are employed for extracting the system-based, lexical, syntactic and semantic characteristics of a word. This results in the total of 25 major feature types as follows:

\footnotetext{
${ }^{3}$ http://www.statmt.org/wmt10/

${ }^{4}$ https://wapiti.limsi.fr/
} 
- Target Side: target word; bigram (trigram) backward sequences; number of occurrences

- Source Side: source word(s) aligned to the target word

- Alignment Context (Nguyen et al. 2011): the combinations of the target (source) word and all aligned source (target) words in the window \pm 2

- Word posterior probability (Ueffing et al., 2003)

- Pseudo-reference (Google Translate): does the word appear in the pseudo reference?

- Graph topology (Luong, Besacier and Lecouteux (2013)): number of alternative paths in the confusion set, maximum and minimum values of posterior probability distribution

- Language model (LM) based: length of the longest sequence of the current word and its previous ones in the target (resp. source) LM. For example, with the target word $w_{i}$ : if the sequence $w_{i-2} w_{i-1} w_{i}$ appears in the target LM but the sequence $w_{i-3} w_{i-2} w_{i-1} w_{i}$ does not, the n-gram value for $w_{i}$ will be 3 .

- Lexical Features: word's Part-Of-Speech (POS) obtained by TreeTagger toolkit $^{5}$ on both source and target sides; sequence of POS of all its aligned source words; POS bigram (trigram) backward sequences; binary lexical features: is the character sequence a punctuation? a proper name? a numerical value?

- Syntactic Features: null link (Xiong et al. 2010); constituent label; depth in the constituent tree

- Semantic Features: number of word senses in WordNet.

In the next step, the word's reference labels (or so-called oracle labels) are initially set by using TERp-A toolkit (Snover, 2008) in one of the following classes: "I" (insertions), "S" (substitutions), "T" (stem matches), "Y" (synonym matches), "P" (phrasal substitutions), "E" (exact matches). "E", "T" and "Y" are then regrouped into "good" class "G", meanwhile the rest ("I", "P" and "S") belongs to "bad" class " $B$ ". We observe that $85 \%$ of the words in our dataset are labeled as "G" and $15 \%$ are labeled as "B". Once having the prediction model, we apply it on the test set $(881 \times 1000$ best $=881000$ sentences $)$ and get needed WCE labels along with confidence probabilities. In terms of F-score, our WCE system gets performance in predicting " $G$ " label of $\mathbf{8 7 . 6 5 \%}$, and " $B$ " label of $\mathbf{4 2 . 2 9 \%}$. The above-mentioned feature types were also used in our English - Spanish WCE System submitted for WMT Quality Estimation shared task and achieved a high rank (first rank in 2013 (Luong et al. 2013) and third rank in 2014 (Luong, Besacier and Lecouteux (2014)). Both WCE and oracle labels will be used in the experiments.

\section{WCE in N-best List Re-ranking}

As stated above, the main goal of this idea is to add WCE-based scores along with existing decoder ones (LM, Translation model, Reordering model, etc.) in order

\footnotetext{
${ }^{5}$ http://www.cis.uni-muenchen.de/ schmid/tools/TreeTagger/
} 
to re-select the best hypothesis among $\mathrm{N}$ candidates. We start by describing our proposed features, followed by experiments and further analysis.

\subsection{Proposed Features}

Since the scores resulting from the WCE system are for words, we have to synthesize them in sentence level scores for integrating with the 14 (already existing) Moses decoder scores. Six new scores are proposed; they include:

- The ratio of number of good words to total number of words (1 score)

- The ratio of number of good nouns (verbs) to total number of nouns (verbs) (2 scores)

- The ratio of number of $n$ consecutive good word sequences to the total number of consecutive word sequences; $n=2, n=3$ and $n=4$ ( 3 scores)

Let us give an example in Figure 2: among the total of 18 words of a given SMT hypothesis, we have 12 labeled as " $G$ "; 7 out of 17 word pairs (bigram) are labeled

as " $G G$ " and 3 out of 16 triples are labeled as "GGG". In this case, some of the above scores can be computed as:

$$
\begin{aligned}
\frac{\# \text { good words }}{\# \text { words }} & =\frac{12}{18}=0.667 \\
\frac{\# \text { good bigrams }}{\# \text { bigrams }} & =\frac{7}{17}=0.4118 \\
\frac{\# \text { good trigrams }}{\# \text { trigrams }} & =\frac{3}{16}=0.1875
\end{aligned}
$$

With the features built on oracle labels, we are able to analyze the upper bound performance. In other words, we can establish an "oracle" setting which is comparable to an interactive case where users would validate a word as "G" or "B" without providing any confidence score.

\subsection{Experiments}

To better examine the impact of the proposed scores, we calculate them not only using our predicted WCE system, but also using an oracle WCE (further called "WCE scores" and "oracle scores", respectively). We experiment with the three following systems:

- BL: Baseline SMT system with 14 above decoder scores

- BL+WCE: Baseline +6 predicted WCE scores

- BL+OR: Baseline +6 oracle WCE scores (simulating an interactive scenario).

The weights assigned to these scores are tuned by a 2-fold cross validation on the test set, using MERT (Och 2003) and MIRA (Watanabe, Suzuki, Tsukada and Isozaki (2007)) methods. More specifically, we split the test set into two equivalent parts: $\mathbf{S} 1$ and $\mathbf{S 2}$, then take $\mathbf{S} \mathbf{1}$ as a development set to optimize the parameters 


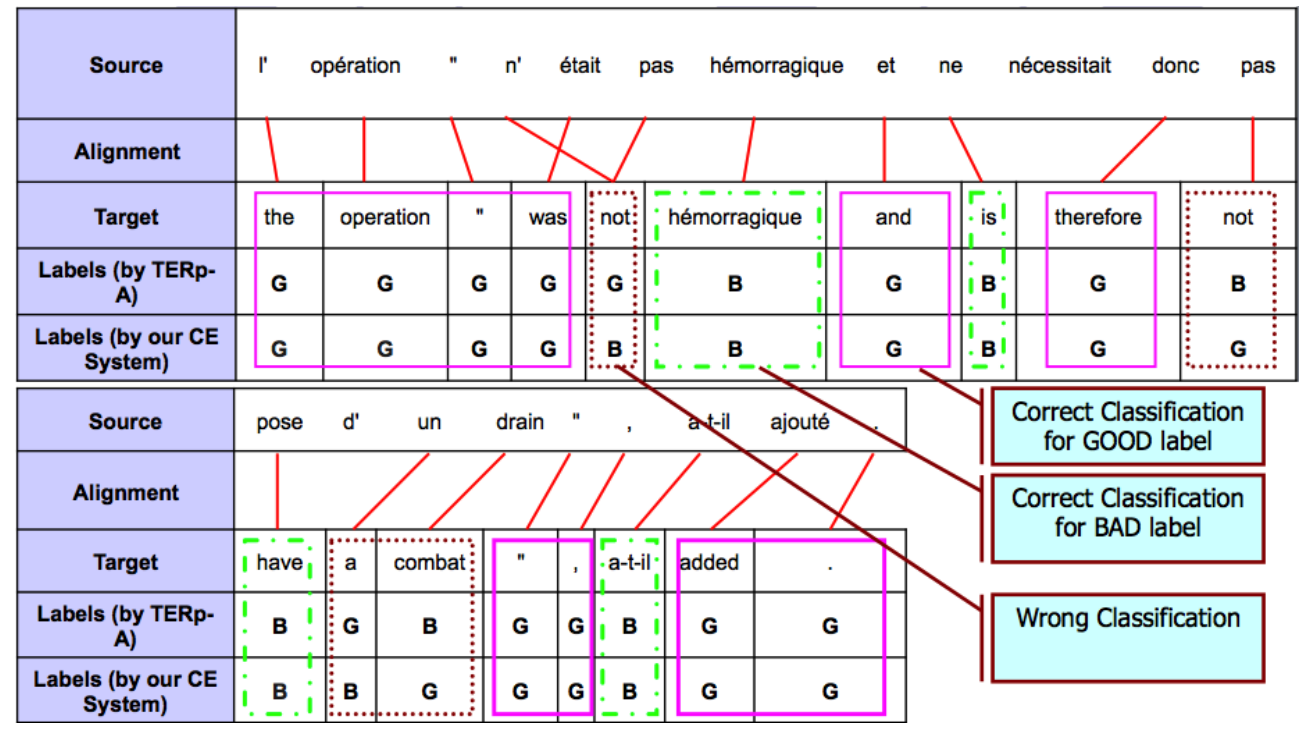

Fig. 2. Example of our WCE classification results for one MT hypothesis

which are then used to decode and re-rank the translations of S2, and vice versa. The translation quality of $\mathbf{B L}, \mathbf{B L}+\mathbf{W C E}$ and $\mathbf{B L}+\mathbf{O R}$ systems are reported in Table 2. The results obtained show that the integration of oracle scores signifi-

\begin{tabular}{lccc|ccc}
\hline \multirow{2}{*}{ Systems } & \multicolumn{3}{c}{ MERT } & \multicolumn{3}{c}{ MIRA } \\
\cline { 2 - 7 } & BLEU & TER & TERp-A & BLEU & TER & TERp-A \\
\hline BL & 52.31 & 0.2905 & 0.3058 & 50.69 & 0.3087 & 0.3036 \\
BL+OR & $\mathbf{5 8 . 1 0}$ & $\mathbf{0 . 2 5 5 1}$ & $\mathbf{0 . 2 5 4 4}$ & $\mathbf{5 5 . 4 1}$ & $\mathbf{0 . 2 7 7 8}$ & $\mathbf{0 . 2 6 8 2}$ \\
BL+WCE & 52.77 & 0.2891 & 0.3025 & 51.01 & 0.3055 & 0.3012 \\
WCE + 25\% & 53.45 & 0.2866 & 0.2903 & 51.33 & 0.3010 & 0.2987 \\
WCE + 50\% & 55.77 & 0.2730 & 0.2745 & 53.63 & 0.2933 & 0.2903 \\
WCE + 75\% & 56.40 & 0.2687 & 0.2669 & 54.35 & 0.2848 & 0.2822 \\
\hline Oracle BLEU score & BLEU $=\mathbf{6 0 . 4 8}$ & & & &
\end{tabular}

Table 2. Translation quality of the baseline system (only decoder scores) and that with additional scores from real "WCE" or "oracle" WCE system

cantly boosts the MT output quality, measured by all three metrics and optimized by both tuning methods employed. We gained 5.79 and 4.72 points in BLEU score, by MERT and MIRA (respectively). With TER, BL+OR helps to gain 0.03 point in both methods. Meanwhile, in case of TERp-A, the improvement is 0.05 point for MERT and 0.03 point for MIRA. It is worthy to mention that the possibility of obtaining such oracle labels is doable through a human-interaction scenario (which could be built from a tool like PET (Post-Editing Tool) (Aziz, De Sousa and Specia (2012)) for instance). In such an environment, once having the hypothesis produced by the first pass (translation task), the human editor could simply 
click on words considered as bad (B), the other words being implicitly considered as correct $(\mathrm{G})$.

For more insightful understanding about WCE scores' role, we calculate the possible optimal BLEU score obtained from the $N$-best list. Applying the sentence-level BLEU+1 metric (Nakov, Guzman and Vogel (2012)) over candidates in the list, we select the one with highest score and aggregate all of them in an oracle-best translation; the resulting performance obtained is $\mathbf{6 0 . 4 8}$. This score shows that the simulated interactive scenario $(\mathbf{B L}+\mathbf{O R})$ is less than optimal only 2.38 points (in case of MERT) and clearly overpasses the baseline (8.17 points below the best score).

The contribution of a real (predicted) WCE system seems more modest: BL+WCE marginally increases BLEU scores of BL (0.46 gain in case of optimizing by MERT and 0.32 by MIRA). For both TER and TERp-A metric, the improvements are also negligible. To verify the significance of this result, we estimate the $p$-value between BLEU of BL+WCE system and BLEU of baseline BL relying on Approximate Randomization (AR) method (Clark, Dyer, Lavie and Smith (2011)) which indicates if the improvement yielded by the optimized system is likely to be generated again by some random processes (randomized optimizers). After various optimizer runs, we randomly selected 5 optimizer outputs to perform the AR test and obtain a $p$-value of $\mathbf{0 . 0 1}$. This result reveals that the improvement yielded by $\mathbf{B L}+\mathbf{W C E}$ is significant although small, originated from the contribution of WCE score, not by any optimizer variance. This modest but positive change in BLEU score using WCE features encourages us to investigate and analyze further about WCE scores' impact by illustrating the gradual improvement of WCE performance, which we now explain. Firstly, we filter out all wrongly classified words in the test set (by matching against the oracle labels) and push them into a temporary set, called $\mathbf{T}$. Then, we correct randomly a percentage $(25 \%, 50 \%$, or $75 \%)$ of labels in $\mathbf{T}$. Finally, the altered $\mathbf{T}$ will be integrated back with the correctly predicted part (by the WCE system) in order to form a new "simulated" result set. This strategy results in three "virtual" WCE systems called "WCE+N\%" (N=25, 50 or 75 ), which use 14 decoder scores (including 7 reordering, 1 language model, 5 translation model and 1 word penalty scores) and 6 "simulated" WCE scores. From each of the above systems, the whole experimental setting is identical to what we did with the original WCE and oracle systems: six scores are built and combined with existing 14 system scores for each hypothesis in the $N$-best list. After that, MERT and MIRA methods are invoked to optimize their weights, and finally the reordering is performed thanks to these scores and appropriate optimal weights. The translation quality measured by BLEU, TER and TERp-A after re-ranking using "WCE+N\%" $(\mathrm{N}=25,50,75)$ can be seen also in Table 2.

We note that all obtained scores behave as expected: the better performance WCE system reaches, the clearer its role in improving MT output quality. Diminishing $25 \%$ of the wrongly predicted words leads to a gain of 0.68 point (by MERT) and 0.32 (by MIRA) in BLEU score. More significant increases of BLEU 3.00 and BLEU 3.63 (MERT) can be achieved when prediction errors decrease up to $50 \%$ 
and $75 \%$. These scores suggest that $\mathrm{WCE}$ has a promising role in improving MT quality if its quality is adequate.

\section{WCE for SMT Search Graph Re-decoding}

The major drawback of the approach previously presented is that the search is limited to a set of $N$ best sentences. What would happen if this space was widened? Will it be efficient for mining better hypothesis? These questions motivate us to investigate SMT search graph re-decoding.

\subsection{Search Graph Structure}

The SMT decoder's Search Graph (SG) can be roughly considered as a "vast warehouse" storing most promising hypotheses generated by the SMT system during decoding for a given source sentence. In this large directed acyclic graph, each hypothesis is represented by a path, carrying all nodes between its start and end ones, along with the edges connecting adjacent nodes. One hypothesis is called complete when all the source words are covered and incomplete otherwise. Starting from the empty initial node, the SG is gradually enlarged by expanding hypotheses during decoding. In order to facilitate the access and the cost calculation, each hypothesis $\mathbf{H}$ is further characterized by the following fields:

- hyp: hypothesis ID

- back: the backpointer pointing to its previous cheapest path.

- transition: the cost to expand from the previous hypothesis (denoted by $\operatorname{pre}(\mathbf{H}))$ to this one.

- score: the cost of this hypothesis: $\operatorname{score}(H)=\operatorname{score}($ pre $(H))+$ transition.

- out: the last output (target) phrase, can contain multiple words.

- covered: the source coverage of out, represented by the start and the end position of the source words translated into out.

Figure 3 illustrates a simple SG generated for the source sentence: "identifier et mesurer les facteurs de mobilization". The attributes "t" and "c" refer to the transition cost and the source coverage, respectively. Hypothesis $\mathbf{1 7 5 5 4 1}$ is extended from $\mathbf{5 7 5 5 2}$, when the three words from 3rd to 5 th position of the source sentence ("les facteurs de") are translated into "the factors of" with the transition cost of -8.5746 . Hence, its cost is: $\operatorname{score}(175541)=\operatorname{score}(57552)+\operatorname{transition}(175541)=$ $-16.1014+(-8.5746)=-24.6760$. Three rightmost hypotheses: 204119, 204109 and $\mathbf{1 9 8 7 2 1}$ are complete since they cover all source words. Among them, the cheapest-cost one is $\mathbf{1 9 8 7 2 1}$, from which the model-best translation is read off by tracing back to the initial node 0: "identify the causes of action .".

\subsection{Overview of the Approach}

We assume that the decoder generates $\mathrm{N}$ best hypotheses $T=\left\{T_{1}, T_{2}, \ldots, T_{N}\right\}$ at the end of the first pass. Using the WCE system (which can only be applied to sequences 


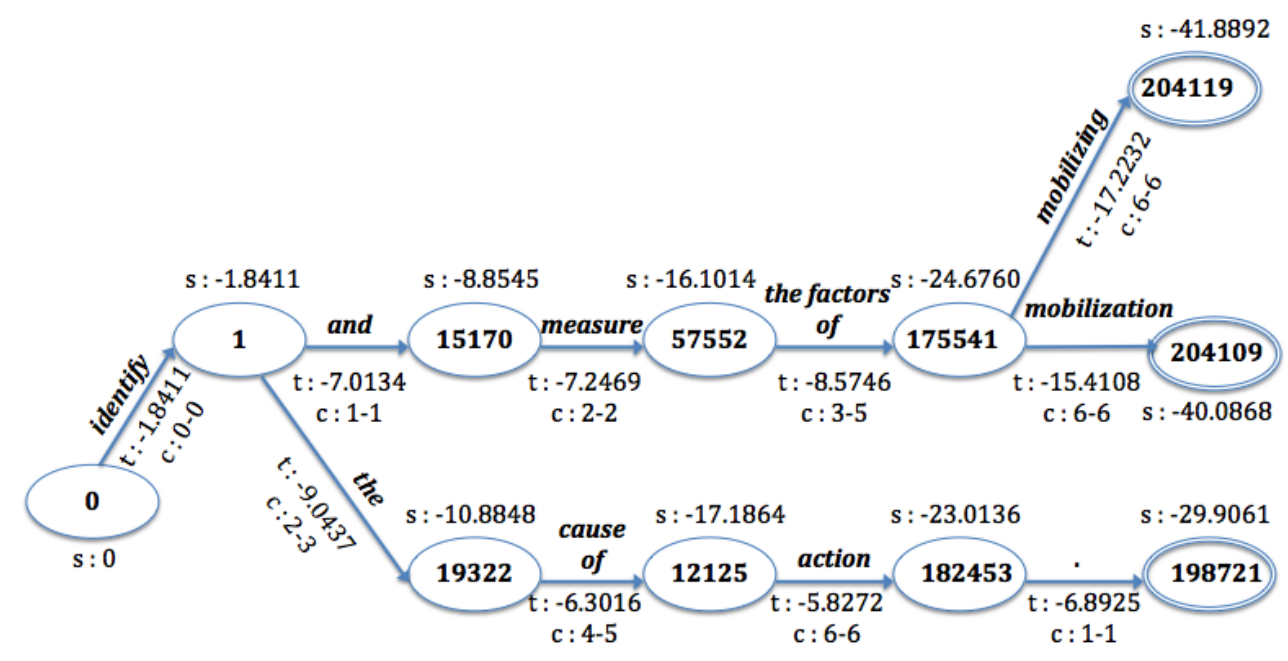

Fig. 3. An example of search graph representation

of words - and not directly to the search graph - that is why $\mathrm{N}$ best hypotheses are used), we are able to assign the $j$-th word in the hypothesis $T_{i}$, denoted by $t_{i j}$, with one appropriate quality label, $c_{i j}$ ( i.e. " $G$ " (Good: no translation error), " $B$ " (Bad: need to be edited)), along with the confidence probabilities $\left(P_{i j}(G), P_{i j}(B)\right.$ or $P(G), P(B)$ for short, where $P(B)=1-P(G))$. Then, the second pass is carried out by considering every word $t_{i j}$ as well as its labels and scores $c_{i j}, P(G), P(B)$. Our principal idea is that, if $t_{i j}$ is a positive (good) translation, i.e. $c_{i j}=$ " $G$ " or $P(G) \approx 1$, all hypotheses $H_{k} \in S G$ containing it in the SG should be "rewarded" by reducing their cost. On the contrary, those containing negative (bad) translation will be "penalized". Let reward $\left(t_{i j}\right)$ and penalty $\left(t_{i j}\right)$ denote the reward or penalty score of $t_{i j}$. The new transition cost of $H_{k}$ after being updated is formally defined by:

$$
\operatorname{transition}^{\prime}\left(H_{k}\right)=\operatorname{transition}\left(H_{k}\right)+ \begin{cases}\operatorname{reward}\left(t_{i j}\right) & \text { if } t_{i j}=\text { good } \\ \operatorname{penalty}\left(t_{i j}\right) & \text { if otherwise }\end{cases}
$$

The update finishes when all words in the $N$-best list have been considered. We then re-compute the new score of complete hypotheses by tracing backward via backpointers and aggregating the transition cost of all their edges. Essentially, the re-decoding pass reorders SG hypotheses: the more " $G$ " words (predicted by WCE system) they contain, the more cost reduction will be made and consequently, the more opportunity they get to be the best hypothesis. It is vital to note that, during the update process, we might face a phenomena that the word $t_{i j}$ (corresponds to the same source words) occurs in different sentences of the $N$-best list. In this case, for the sake of simplicity, we process it only at its first occurrence (in the highest rank sentence) instead of updating the hypotheses containing it multiple times. In other words, if we meet the word $t_{i j}$ which aligns to the same source word once 
again in the next N-best sentence(s), no further score update will be done in the SG.

\subsection{Update Score Definitions}

Defining the update scores is a nontrivial issue as there is no quantitative estimate on the importance of WCE scores over the SG scores or vice versa. Our approach is to measure their importance empirically via weight optimization. In this article, we propose several types of update scores, deriving from the global or local cost.

\subsubsection{Definition \#1: Global Update Score}

In this type, an unique score derived from the cost of the current best hypothesis $H^{*}$ (by the first pass) is used for all updates. We propose to compute this score by two ways: (a) exploiting WCE labels $\left\{c_{i j}\right\}$; or (b) only WCE confidence probabilities $\{P(G), P(B)\}$ will matter and WCE labels will be left aside.

Definition \#1a:

$$
\begin{array}{r}
\operatorname{penalty}\left(t_{i j}\right)=\alpha * \frac{\operatorname{score}\left(H^{*}\right)}{\# \operatorname{words}\left(H^{*}\right)} \\
\operatorname{reward}\left(t_{i j}\right)=-\operatorname{penalty}\left(t_{i j}\right)
\end{array}
$$

Where \#words $\left(H^{*}\right)$ is the number of target words in $H^{*}$, the positive coefficient $\alpha$ accounts for the impact level of this score on the final cost of the hypothesis and can be optimized during experiments. Here, penalty $\left(t_{i j}\right)$ gets negative sign (since $\left.\operatorname{score}\left(H^{*}\right)<0\right)$ and will be added to the transition cost of all hypotheses containing $t_{i j}$ in case where this word is labelled as " $B$ "; whereas $\operatorname{reward}\left(t_{i j}\right)$ (same value, opposite sign) is used in the other case.

Definition \#1b:

$$
\begin{array}{r}
\text { update }\left(t_{i j}\right)=\alpha * P(B) * \frac{\operatorname{score}\left(H^{*}\right)}{\# \operatorname{words}\left(H^{*}\right)}-\beta * P(G) * \frac{\operatorname{score}\left(H^{*}\right)}{\# \operatorname{words}\left(H^{*}\right)} \\
=(\alpha * P(B)-\beta * P(G)) * \frac{\operatorname{score}\left(H^{*}\right)}{\# \operatorname{words}\left(H^{*}\right)}
\end{array}
$$

Where $P(G), P(B)(P(G)+P(B)=1)$ are the probabilities of "Good" and "Bad" class of $t_{i j}$. The positive coefficients $\alpha$ and $\beta$ can be tuned in the optimization phase. In this definition, the fact that update $\left(t_{i j}\right)$ is a reward $\left(\operatorname{reward}\left(t_{i j}\right)\right)$ or a penalty (penalty $\left.\left(t_{i j}\right)\right)$ will depend on $t_{i j}$ 's goodness. Indeed, we have: update $\left(t_{i j}\right)=$ $\operatorname{reward}\left(t_{i j}\right)$ if update $\left(t_{i j}\right)>0$, which means: $\alpha *[1-P(G)]-\beta * P(G)<0$ (since score $\left.\left(H^{*}\right)<0\right)$, therefore $P(G)>\frac{\alpha}{\alpha+\beta}$.

On the contrary, if $P(G)$ is under this threshold, update $\left(t_{i j}\right)$ takes a negative value and therefore becomes a penalty.

\subsubsection{Definition \#2: Local Update Score}

The update score of each (local) hypothesis $H_{k}$ depends on its current transition cost, even when they cover the same word $t_{i j}$. Similarly to Definition 1, two sub- 
types are defined as follows:

Definition \#2a:

$$
\operatorname{penalty}\left(t_{i j}\right)=-\operatorname{reward}\left(t_{i j}\right)=\alpha * \operatorname{transition}\left(H_{k}\right)
$$

Definition \#2b:

$$
\begin{array}{r}
\text { update }\left(t_{i j}\right)=\alpha * P(B) * \operatorname{transition}\left(H_{k}\right)-\beta * P(G) * \operatorname{transition}\left(H_{k}\right) \\
=(\alpha * P(B)-\beta * P(G)) * \operatorname{transition}\left(H_{k}\right)
\end{array}
$$

Where transition $\left(H_{k}\right)$ denotes the current transition cost of hypothesis $H_{k}$, and the meanings of coefficient $\alpha$ (Definition 2a) or $\alpha, \beta$ (Definition 2b) are analogous to those of Definition 1a (Definition 1b), respectively.

\subsection{Re-decoding Algorithm}

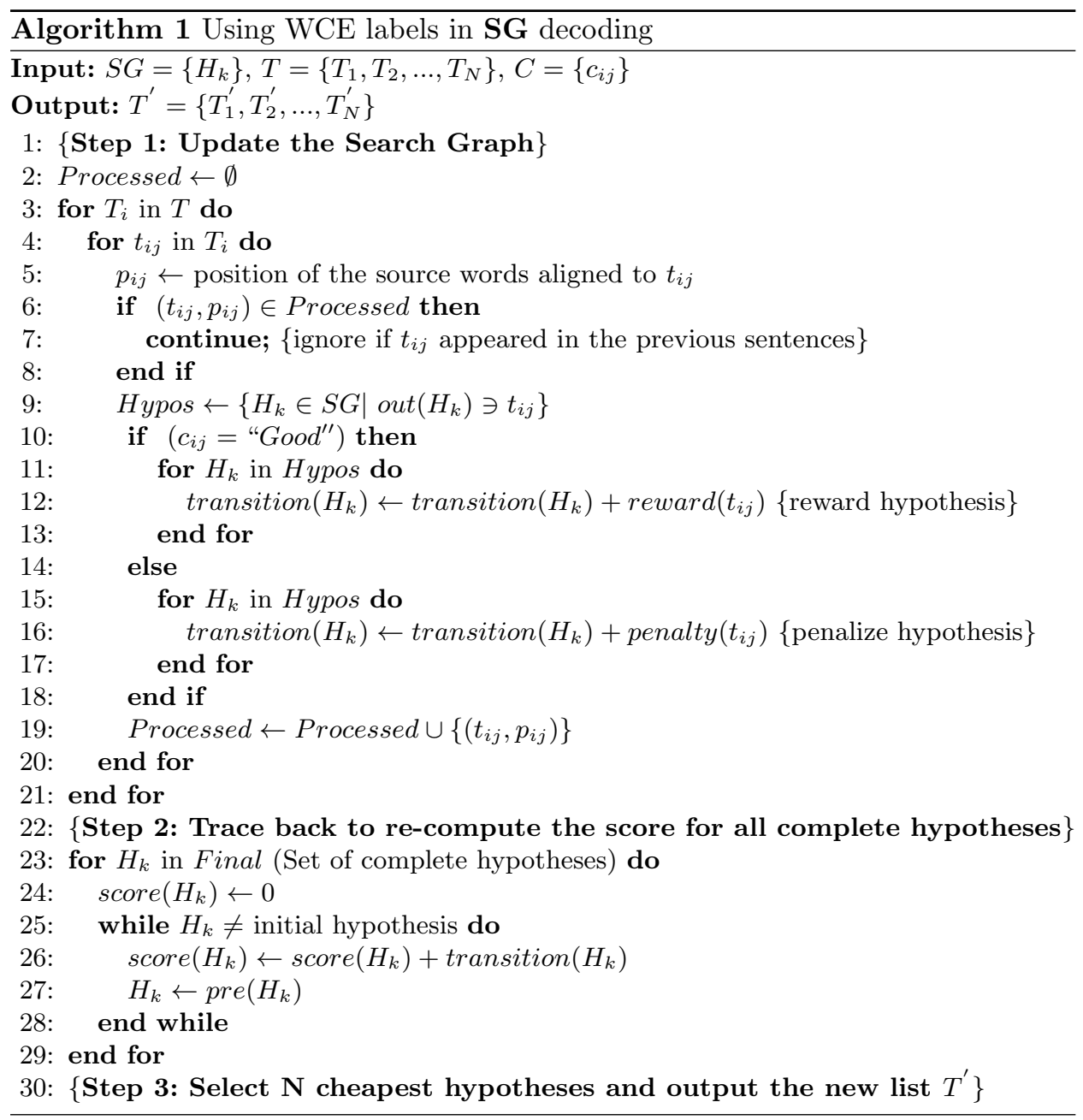


The above pseudo-code depicts our re-decoding algorithm using WCE labels (Definition 1a and Definition 2a). The algorithm in case of using WCE confidence probabilities (Definition $\mathbf{1 b}$ and Definition $\mathbf{2 b}$ ) is essentially similar, except the update step (from line 10 to line 18) is replaced by the following part:

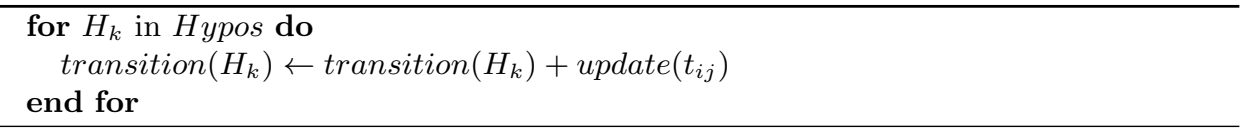

During the update process, the pairs including the visited word $t_{i j}$ and the position of its aligned source words $p_{i j}$, are consequently admitted to be Processed, so that all the analogous pairs $\left(t_{i j}^{\prime}, p_{i j}^{\prime}\right)$ occuring in the latter sentences can be discarded. For each $t_{i j}$, we search for all hypotheses in the SG whose the output phrase (stored in the field out) contains $t_{i j}$ to form the set Hypo. To avoid the ambiguity where $t_{i j}$ occurs multiple times in the sentence, the position coverage (stored in the field covered) must be ensured to bound the position of the aligned source word of the current $t_{i j}$. Then, the confidence score of $t_{i j}$, denoted by $c_{i j}$ (or $P(G)$ ), determines whether all members $H_{k}$ in Hypo will be rewarded or penalized. Once having all words in the $N$-best list visited, we obtain a new SG with updated transition costs for all edges containing them. On this updated SG, we re-compute the score of all complete hypotheses stored in Final. Finally, we backtrack the cheapest-cost hypothesis to obtain the new translation.

\begin{tabular}{ccccccccc}
\hline Rank & Cost & \multicolumn{6}{c}{ Hypothese + WCE labels } \\
\hline $\mathbf{1}$ & -29.9061 & identify & the & cause & of & action & . \\
& & G & G & G & G & B & B & \\
\hline $\mathbf{2}$ & -40.0868 & identify & and & measure & the & factors & of mobilization \\
& & G & G & G & G & G & G & G \\
\hline
\end{tabular}

Table 3. The N-best (N=2) list generated by the SG in Figure 3 and WCE labels

These above depictions can be clarified by taking another look at the example in Figure 3: from this SG, the $N$-best list (for the sake of simplicity, we choose $N=2$ ) is generated as the single-pass decoder's result. According to our approach, the second pass starts by tagging all words in the list with their confidence labels, as seen in Table 3. Then, the graph update process is performed for each word in the list, sentence by sentence, which details are tracked in Figure 4. In this example, we apply Definition 1a to calculate the reward or penalty score, with $\alpha=\frac{1}{2}$, resulting in: penalty $\left(t_{i j}\right)=-\operatorname{reward}\left(t_{i j}\right)=\frac{1}{2} * \frac{-29.9061}{6}=-2.4922$. Firstly, all hypotheses containing words in the 1st ranked sentence are considered. Since the word "identify" is labeled as " $G$ ", its corresponding edge (connecting two nodes $\mathbf{0}$ and $\mathbf{1})$ is rewarded: $t_{\text {new }}=t_{\text {old }}+$ reward $=-1.8411+2.4922=+0.6511$. On the contrary, the edge between two nodes 121252 and 182453 is penalized and takes new cost: $t_{\text {new }}=t_{\text {old }}+$ penalty $=-5.8272+(-2.4922)=-8.3194$, due to 


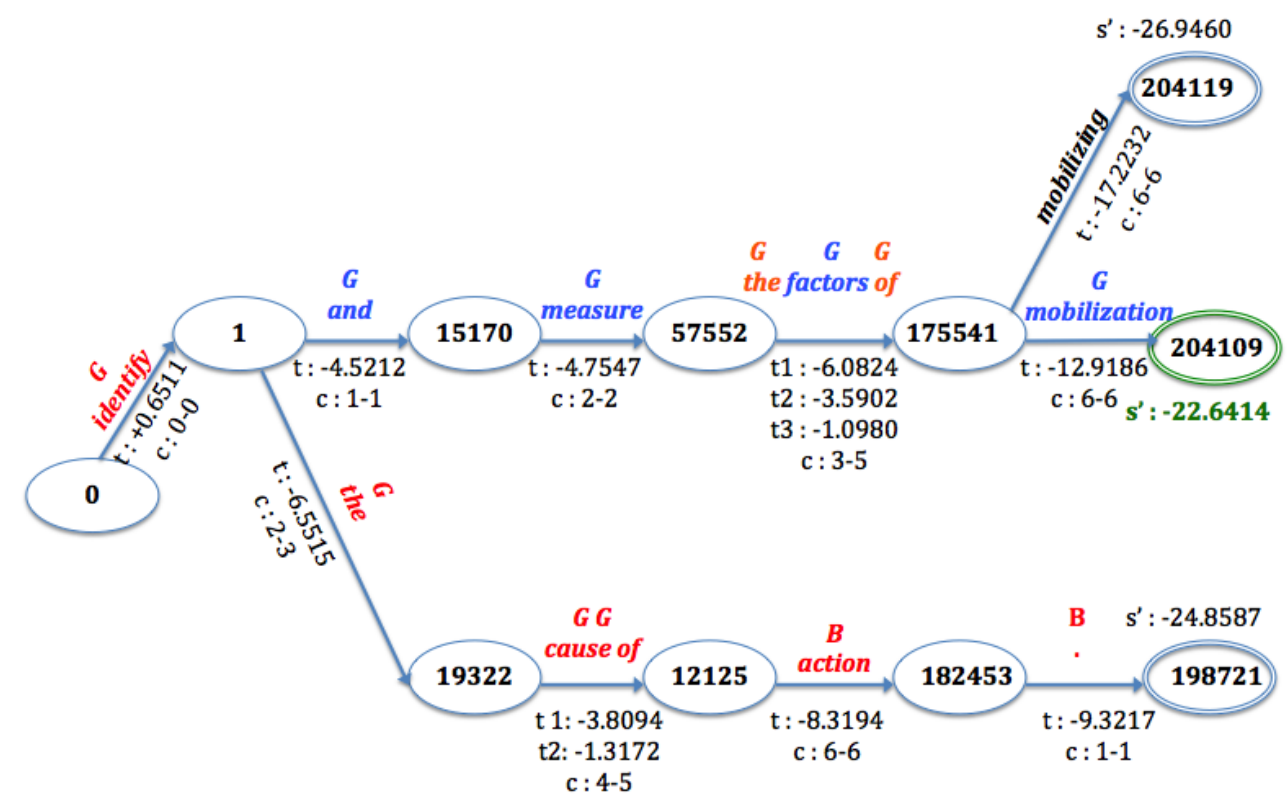

Fig. 4. Details of update process for the SG in Figure 3. The first loop is represented in red color, while the second one is in blue. For edges with multiple updates, all transition costs after each update are logged. The winning cost is emphasized by green color.

the bad quality of the word "action". The edges having multiple considered words (e.g. between nodes 19322 and $\mathbf{1 2 1 2 5 2}$ ) will be updated multiple times, and the transition costs after each update can be also observed in Figure 4 ( e.g. $t 1, t 2$, etc). Next, when the 2nd-best is taken into consideration, all repeated words (e.g. "identify", "the" and "of") are ignored since they have been visited before, whereas the remaining ones are identically processed. The only untouched edge in this SG corresponds to the word "mobilizing", as this word does not belong to the list. Once having the update process finished, we recalculate the final cost for every complete path and return the new best translation: "identify and measure the factors of mobilization" (new cost $=-22.6414$ ).

\subsection{Experimented Decoders}

As in the Re-ranking approach, we investigate the WCE's contributions in two scenarios: predicted WCE and ideal WCE (oracles). We experiment with the seven following decoders:

- BL: Baseline (1-pass decoder)

- BL+WCE(1a, 1b, 2a, 2b): four 2-pass decoders, using our estimated WCE labels and confidence probabilities to update the SGs, and the update scores are calculated by Definition (1a, 1b, 2a, 2b). 
- BL+OR(1a, 2a): two 2-pass decoders, computing the reward or penalty scores by Definition (1a, 2a) on the oracle labels

It is important to note that, when using oracle labels, Definition $\mathbf{1 b}$ becomes Definition 1a and Definition $\mathbf{2 b}$ becomes Definition 2a, since if a word $t_{i j}$ is labelled as "G", then $P(G)=1$ and $P(B)=0$, and vice versa. In order to tune the coefficients $\alpha$ and $\beta$, we carry out a $\mathbf{2}$-fold cross validation on the test set. First, the set is split into two equivalent parts: S1 and S2. Playing the role of a development set, $\mathbf{S} \mathbf{1}$ will train the parameters which are then used to compute the update scores on $\mathbf{S 2}$ re-decoding process, and vice versa. The optimization steps are handled by CONDOR toolkit (Frank 2004), in which we vary $\alpha$ and $\beta$ within the interval $[0.00 ; 5.00]$. Test set is further divided to launch experiments in parallel on our cluster using an open-source batch scheduler: OAR (Capit and Joseph 2013). This mitigates the overall processing times on such huge SGs. The re-decoding results for them are properly concatenated for evaluation. Tuned values for these parameters are: $\alpha=0.9$ (Definition 1a); $\alpha=1.7, \beta=0.8$ (Definition 1b); $\alpha=0.9$ (Definition 2a); and $\alpha=1.8, \beta=0.8$ (Definition 2b).

\section{Results}

\begin{tabular}{lcccccccc}
\hline Systems & \multicolumn{3}{c}{ Performance } & \multicolumn{3}{c}{ Comparison to BL } & $\boldsymbol{p}$ - \\
& BLEU $\uparrow$ & TER $\downarrow$ & TERp-A $\downarrow$ & B (\%) & E (\%) & W (\%) & value \\
\hline BL & 52.31 & 0.2905 & 0.3058 & - & - & - & - \\
BL+WCE(1a) & $\mathbf{5 3 . 8 0}$ & $\mathbf{0 . 2 8 7 6}$ & $\mathbf{0 . 2 9 2 2}$ & 28.72 & 57.43 & 13.85 & 0.00 \\
BL+WCE(1b) & 53.24 & 0.2896 & 0.2995 & 26.45 & 59.26 & 14.29 & 0.00 \\
BL+WCE(2a) & 53.32 & 0.2893 & 0.3018 & 23.68 & 60.11 & 16.21 & 0.02 \\
BL+WCE(2b) & 53.07 & 0.2900 & 0.3006 & 22.27 & 55.17 & 22.56 & 0.01 \\
BL+OR(1a) & $\mathbf{6 0 . 1 8}$ & $\mathbf{0 . 2 2 9 8}$ & $\mathbf{0 . 2 2 6 4}$ & 62.52 & 24.36 & 13.12 & - \\
BL+OR(2a) & 59.98 & 0.2340 & 0.2355 & 60.18 & 28.82 & 11.00 & - \\
\hline
\end{tabular}

Table 4. Translation quality of the conventional decoder and the 2-pass decoders using scores from predicted or oracle WCE, followed by the percentage of better $(B)$, equivalent $(E)$ or worse $(W)$ sentences compared to $\boldsymbol{B L}$ system, as well as p-values

Table 4 shows the translation performances of all experimental decoders and their percentages of sentences which outperform, remain equivalent or degrade the baseline hypotheses (when match against the references, measured by TER). Results suggest that using oracle labels to re-direct the graph searching considerably boosts the baseline quality. BL+OR(1a) augments 7.87 points in BLEU, and diminishes 0.0607 (0.0794) point in TER (TERp-A), compared to BL. Meanwhile, with $\mathbf{B L}+\mathbf{O R}(\mathbf{2 a})$, these gains are 7.67, 0.0565 and 0.0514 (in that order). Besides, the contribution of our WCE system scores seems less prominent, yet positive: the best performing BL+WCE(1a) increases 1.49 BLEU points of BL $(0.0029$ and 0.0136 gained for TER and TERp-A). The small $\boldsymbol{p}$-values (in the range [0.00; 0.02], seen on Table 2) estimated between BLEU of each BL+WCE system and that 
of BL using Approximate Method ( Clark et al. 2011) indicate that these performance improvements are significant. Results also reveal that the use of WCE labels is slightly more beneficial than that of confidence probabilities: $\mathbf{B L}+\mathbf{W C E}(\mathbf{1 a})$ and $\mathbf{B L}+\mathbf{W C E}(\mathbf{2 a})$ outperform $\mathbf{B L}+\mathbf{W C E}(\mathbf{1 b})$ and $\mathbf{B L}+\mathbf{W C E}(\mathbf{2 b})$. In both scenarios, we observe that the global update score (Definition 1) performs better compared to the local one (Definition 2). For more insightful understanding about WCE scores' usefulness, we make a comparison with the best achievable hypotheses in the SG (oracles), based on the "LM Oracle" approximation approach presented in (Sokolov, Wisniewski and Yvon (2012)). This method simplifies the oracle decoding to the problem of searching for the cheapest path on a SG where all transition costs are replaced by the $n$-gram LM scores of the corresponding words. The LM is built for each source sentence uniquely using its target post-edition. We update the SG by assigning all edges with the LM back-off score of the word it contains. Finally, we combine the oracles of all sentences yielding BLEU oracle of 66.48: 6.30 points higher than $\mathbf{B L}+\mathbf{O R}(\mathbf{1 a})$. This result reveals that the use of oracle WCE scores, although helps to outperform significantly over the Baseline (52.31 BLEU

$\rightarrow 60.18 \mathrm{BLEU})$, yet still remains a big gap to the best-achievable performance by SG re-decoding. It opens a room for future work on how to exploit WCE scores more efficiently toward this goal.

We close this section with some examples of outputs before and after the redecoding process (shown in Table 5). In the first example, re-decoding yields a slightly better translation compared to the baseline MT system. Using labels predicted by WCE: "Bad" for "a" (at the beginning of the MT hypothesis) and "penalty", and "Good" for the rest, BL+WCE(1a) led to a new candidate whose last words ("demoralization death") are closer to the post-edition ("deadly demoralization") than the corresponding part of BL ("penalty demoralisation"). Similarly, in the second example, thanks to word error detection in the second example (tagged for "it has", "speech that was", and "post route"), $\mathbf{B L}+\mathbf{O R}(\mathbf{1 a})$ helped to translate correctly the pronoun ( "he" instead of "it"), as well as to translate better the final part of the source sentence (into "after operating were normal"). The positive contributions of WCE-predicted labels and scores can also be observed in the remaining (from the third to the fifth) examples in the same table.

\section{Discussion, Conclusion and Perspectives}

If we compare the performances between the use of WCE for $N$-best list re-ranking (Table 2) and SG Re-decoding (Table 4), the results suggest that the contribution of WCE in SG re-decoding outperforms that in N-best re-ranking in both "oracle" or real scenarios. BL+OR(1a) outperforms its corresponding oracle reranker BL+OR(N-best_Reranking) in 2.08 points of BLEU, diminishes 0.0253 (0.0280) in TER (TERp-A). Meanwhile, BL+WCE(1a) beats BL+WCE(Nbest_Reranking) (the re-ranker which uses predicted WCE scores) in 1.03 (BLEU), 0.0015 (TER), 0.0103 (TERp-A). In addition, there are 178 out of 881 sentences $(20.20 \%)$ outputted by BL+WCE(1a) that do not belong to the N-best list, suggesting that the re-decoding method can help to generate candidates which 


\begin{tabular}{|c|c|}
\hline \multicolumn{2}{|l|}{ Example 1 } \\
\hline Source & $\begin{array}{l}\text { une démobilisation des employés peut déboucher sur une démoralisation } \\
\text { mortifère }\end{array}$ \\
\hline BL & a demobilisation employees can lead to a penalty demoralisation \\
\hline $\mathrm{BL}+\mathrm{WCE}(\mathbf{1 a})$ & a demobilisation of employees can lead to a demoralization death \\
\hline Post-edition & demobilization of employees can lead to a deadly demoralization \\
\hline \multicolumn{2}{|l|}{ Example 2} \\
\hline Source & $\begin{array}{l}\text { celui-ci a indiqué que l'intervention s'était parfaitement bien déroulée } \\
\text { et que les examens post-opératoires étaient normaux }\end{array}$ \\
\hline BL & $\begin{array}{l}\text { it has indicated that the speech that was well conducted, and that } \\
\text { the tests were normal post route }\end{array}$ \\
\hline BL+OR (1a) & $\begin{array}{l}\text { he indicated that the intervention is very well done, and that the } \\
\text { tests after operating were normal }\end{array}$ \\
\hline Post-edition & $\begin{array}{l}\text { he indicated that the operation went perfectly well and the post- } \\
\text { operative tests were normal }\end{array}$ \\
\hline \multicolumn{2}{|l|}{ Example 3} \\
\hline Source & pour le système des transports ferroviaires le s21 est néfaste . \\
\hline BL & for the rail traffic system, s21 is damaging \\
\hline $\mathrm{BL}+\mathrm{OR}(\mathbf{2 a})$ & for the system of rail transport the $\mathrm{s} 21$ is harmful \\
\hline Post-edition & for the railway transportation system the $\mathrm{s} 21$ is harmful \\
\hline \multicolumn{2}{|l|}{ Example 4} \\
\hline Source & $\begin{array}{l}\text { tout comme le rêve, la psychose ouvre les écluses à une marée d' idées } \\
\text { et de fantasmes issue des couches plus profondes de la conscience. }\end{array}$ \\
\hline BL & $\begin{array}{l}\text { as the dream, the hype opens the floodgates to a tide of ideas and } \\
\text { fantasies, the deeper layers of conscience. }\end{array}$ \\
\hline BL+OR (1b) & $\begin{array}{l}\text { like the dream, a psychosis also opens the floodgates to a tide of ideas } \\
\text { and phantasies, which stem from the deepest layers of conscience. }\end{array}$ \\
\hline Post-edition & $\begin{array}{l}\text { just like the dream, the psychosis opens the floodgates to a tide of ideas } \\
\text { and fantasies that emerged from the deepest layers of consciousness . }\end{array}$ \\
\hline \multicolumn{2}{|l|}{ Example 5} \\
\hline Source & $\begin{array}{l}\text { burda avait fait savoir jeudi dernier qu' il se retirait du poste } \\
\text { président-directeur-général dès le mois de janvier et que son successeur } \\
\text { était paul-bernhard kallen. }\end{array}$ \\
\hline BL & $\begin{array}{l}\text { burda said last thursday that it is pulling out of the post président- } \\
\text { directeur-général in january and that his successor was paul-bernhard } \\
\text { kallen. }\end{array}$ \\
\hline $\mathrm{BL}+\mathrm{OR}(2 \mathrm{~b})$ & $\begin{array}{l}\text { burda let it be known last thursday that he is pulling out of his } \\
\text { post président-directeur-général in january and that his successor was } \\
\text { paul-bernhard kallen. }\end{array}$ \\
\hline Post-edition & $\begin{array}{l}\text { burda let it be known last thursday that he is pulling out of his post } \\
\text { as president director general beginning in january and that his successor } \\
\text { would be paul-bernhard kallen. }\end{array}$ \\
\hline
\end{tabular}

Table 5. Examples of MT outputs before and after re-decoding

have not been nominated by SMT decoder. More notably, among these newlyproduced translations, $113(63.5 \%)$ translations are better than the corresponding 1-best of the N-best list, measured by sentence-level BLEU+1 metric (Nakov, Guzman and Vogel (2012)). These positive results can be explained by the following facts: (1) in re-ranking, WCE scores are integrated at sentence level, so word translation errors are not fully penalized; and (2) in re-ranking, best translation 
selection is limited to the $N$-best list, whereas in re-decoding, we allow the search over the entire updated SG (on which not only N-best list paths but also those containing at least one word in this list are altered).

To conclude, we have presented two novel two-pass decoding methods for enhancing SMT quality. From the output of the first pass ( $N$-best list), we predict words' labels and confidence probabilities, then employ them to seek a more valuable candidate; first among the other in the $N$-best list, and then over the entire SGs. In both methods, while "oracle" WCE labels substantially improve the MT quality (to reach the oracle score), real WCE achieves also the positive and promising gains. These methods show that error prediction using WCE helps to guide the decoding to less erroneous parts of the graph.

As future work, we plan to focus on enhancing our WCE system using more linguistic features as well as advanced techniques (feature selection, Boosting method...). We also focus on breaking down the translation errors (i.e. "Bad" label) into more concrete categories, so that they will be more informative. Besides, the update scores used in this article will be further considered towards the consistency with SMT graph scores to obtain a better updated SG. Also, currently in the graph-redecoding process, our WCE scores can only be applied to sequences of words - and not directly to the search graph. Applying WCE to tag directly the search graph will be part of future work. Finally, we will investigate the use of WCE labels to re-decode speech translation graphs and improve spoken language translation (SLT) performance.

\section{References}

Aziz, W., De Sousa, S.C.M., and Specia, L. 2012. Pet: a tool for post-editing and assessing machine translation. In Proceedings of the Eight International Conference on Language Resources and Evaluation (LREC'12), Istanbul, Turkey.

Bicici, E. 2013. Referential translation machines for quality estimation. In Proceedings of the Eighth Workshop on Statistical Machine Translation, 343-351, Sofia, Bulgaria.

Blackwood, Graeme. 2010. Lattice Rescoring Methods for Statistical Machine Translation. $\mathrm{PhD}$ thesis, University of Cambridge.

Blatz, J., Fitzgerald, E., Foster, G., Gandrabur, S., Goutte, C., Kulesza, A., Sanchis, A. and Ueffing, N. 2003. Confidence Estimation for Machine Translation. Technical report, JHU/CLSP Summer Workshop.

Blatz, J., Fitzgerald, E., Foster, G., Gandrabur, S., Goutte, C., Kulesza, A., Sanchis, A. and Ueffing, N. 2004. Confidence Estimation for Machine Translation. In Proceedings of COLING 2004, 315-21, Geneva.

Bojar, O., Buck, C., Callison-Burch, C., Federmann, C., Haddow, B., Koehn, P., Monz, C., Post, M., Soricut, R. and Specia, L. 2013. Findings of the 2013 Workshop on Statistical Machine Translation. In Proceedings of the Eighth Workshop on Statistical Machine Translation, 1-44, Sofia, Bulgaria.

Camargo-de-Souza, J.G., González-Rubio, J., Buck, C., Turchi, M., and Negri, M. 2014. Fbk-upv-uedin participation in the wmt14 quality estimation shared-task. In Proceedings of the Ninth Workshop on Statistical Machine Translation, Baltimore, Maryland, USA.

Capit, N. and Joseph, E. 2013. OAR Documentation - User Guide. LIG laboratory, Laboratoire d'Informatique de Grenoble, France. 
Clark, J., Dyer, C., Lavie, A. and Smith, N. 2011. Better hypothesis testing for statistical machine translation: Controlling for optimizer instability. In Proceedings of the Association for Computational Lingustics.

Duh, K. and Kirchhoff, K. 2008. Beyond log-linear models: Boosted minimum error rate training for n-best re-ranking. In Association for Computer Linguistics (Short Papers), $37-40$.

Felice, M. and Specia, L. 2012. Linguistic Features for Quality Estimation. In Proceedings of the 7th Workshop on Statistical Machine Translation, pp. 96-103, Montreal, Canada.

Frank, V.B. 2004. CONDOR: a constrained, non-linear, derivative-free parallel optimizer for continuous, high computing load, noisy objective functions. PhD thesis, University of Brussels (ULB - Université Libre de Bruxelles), Belgium, 2004.

Gandrabur, S. and Foster, G. 2003. Confidence Estimation for Text Prediction. In Conference on Natural Language Learning (CoNLL), 315-21, Edmonton.

Han, A.L.F., Lu, J., Wong, D.F., Chao, L.S., He, L. and Xing, J. 2013. Quality estimation for machine translation using the joint method of evaluation criteria and statistical modeling. In Proceedings of the Eighth Workshop on Statistical Machine Translation, 365-72, Sofia, Bulgaria.

Kirchhoff, K. and Yang, M. 2005. Improved language modeling for statistical machine translation. In Proceedings of the ACL Workshop on Building and Using Parallel Texts, 125-8, Ann Arbor, Michigan.

Koehn, P., Hoang, H., Birch, A., Callison-Burch, C., Federico, M., Bertoldi, N., Cowan, B., Shen, W., Moran, C., Zens, R., Dyer, C., Bojar, O., Constantin, A. and Herbst, E. 2007. Moses: Open source toolkit for statistical machine translation. In 45th Annual Meeting of the Association for Computational Linguistics, 177-80, Prague, Czech Republic.

Kreutzer, J., Schamoni, S., and Riezler, S. 2015. QUality Estimation from ScraTCH (QUETCH): Deep Learning for Word-level Translation Quality Estimation. In Proceedings of the Tenth Workshop on Statistical Machine Translation, pages 316-22, Lisboa, Portugal. Association for Computational Linguistics.

Lafferty, J., McCallum, A. and Pereira, F. 2001. Conditional Random Fields: Probabilistic Models for Segmenting and Labeling Sequence Data. In Proceedings of the Eighteenth International Conference on Machine Learning, 282-9, San Francisco, CA.

Lavergne, T., Cappé, O. and Yvon, F. 2010. Practical Very Large Scale CRFs. In 48th Annual Meeting of the Association for Computational Linguistics, 504-13, Uppsala, Sweden.

Logacheva, V., Hokamp, C., and Specia, L. 2015. Data enhancement and selection strategies for the word-level Quality Estimation. In Proceedings of the Tenth Workshop on Statistical Machine Translation, pages 330-5, Lisboa, Portugal. Association for Computational Linguistics.

Luong, N.Q. 2012. Integrating Lexical, Syntactic and System-based Features to Improve Word Confidence Estimation in SMT. In Proceedings of JEP-TALN-RECITAL, 43-56, Grenoble, France.

Luong, N.Q., Besacier, L. and Lecouteux, B. 2013. Word confidence estimation and its integration in sentence quality estimation for machine translation. In Proceedings of The Fifth International Conference on Knowledge and Systems Engineering, Hanoi, Vietnam.

Luong, N. Q., Besacier, L. and Lecouteux, B. 2014. LIG System for Word level WE Task at WMT14. In Proceedings of the Ninth Workshop on Statistical Machine Translation, Baltimore, Maryland, USA.

Luong, N.Q., Lecouteux, B. and Besacier, L. 2013. LIG System for WMT13 QE Task: Investigating the Usefulness of Features inWord Confidence Estimation for MT. In Proceedings of the Eighth Workshop on Statistical Machine Translation, Sofia, Bulgaria.

Nakov, P., Guzman, F. and Vogel, S. 2012. Optimizing for sentence-level bleu+1 yields short translations. In Proceedings of COLING 2012, 1979-94, Mumbai, India. 
Nguyen, B., Huang, F. and Al-Onaizan, Y. 2011. Goodness: A Method for Measuring Machine Translation Confidence. In Proceedings of the 49th Annual Meeting of the Association for Computational Linguistics, 211-9, Portland, Oregon.

Och, F.J. 2003. Minimum error rate training in statistical machine translation. In Proceedings of the 41st Annual Meeting of the Association for Computational Linguistics, 160-67, Sapporo, Japan.

Papineni, K., Roukos, S., Ard, T. and Zhu, W.J. 2002. Bleu: a method for automatic evaluation of machine translation. In Proceedings of the 40th Annual Meeting of the Association for Computational Linguistics.

Petrov, S. and Klein, D. 2007. Improved inference for unlexicalized parsing. In Proceedings of NAACL HLT 200\%, 404-11, Rochester, NY.

Potet, M., Rodier, E.E., Besacier, L. and Blanchon, H. 2012. Collection of a Large Database of French-English SMT Output Corrections. In 8th International Conference on Language Resources and Evaluation, Istanbul.

Raybaud, S., Langlois, D., and Smaïli, K. 2011. "This sentence is wrong." Detecting errors in machine - translated sentences. Machine Translation, 25(1):1-34.

Shah, K., Logacheva, V., Paetzold, G., Blain, F., Beck, D., Bougares, F., and Specia, L. 2015. SHEF-NN: Translation Quality Estimation with Neural Networks. In Proceedings of the Tenth Workshop on Statistical Machine Translation, pages 342-7, Lisboa, Portugal. Association for Computational Linguistics.

Shang, L., Cai, D., and Ji, D. 2015. Strategy- Based Technology for Estimating MT Quality. In Proceedings of the Tenth Workshop on Statistical Machine Translation, pages 348-52, Lisboa, Portugal. Association for Computational Linguistics.

Snover, M., Madnani, N., Dorr, B. and Schwartz, R. 2008. Terp system description. In MetricsMATR workshop at the Conference of the Association for Machine Translation in the Americas (AMTA).

Sokolov, A., Wisniewski, G. and Yvon, F. 2012. Computing lattice bleu oracle scores for machine translation. In Proceedings of the 13th Conference of the European Chapter of the Association for Computational Linguistics, 120-9, Avignon, France.

Sokolov, A., Wisniewski, G. and Yvon, F. 2012. Non-linear n-best list reranking with few features. In Proceedings of the Conference of the Association for Machine Translation in the Americas (AMTA).

Soricut, R. and Echihabi, A. 2010. Trustrank: Inducing Trust in Automatic Translations via Ranking. In Proceedings of the 48th Annual Meeting of the Association for Computational Linguistics, 612-21, Uppsala, Sweden.

Stolcke, A. 2002. Srilm - an Extensible Language Modeling Toolkit. In 7th International Conference on Spoken Language Processing, 901-4, Denver, USA.

Tezcan, A., Hoste, V., Desmet, B., and Macken, L. 2015. UGENT-LT3 SCATE System for Machine Translation Quality Estimation. In Proceedings of the Tenth Workshop on Statistical Machine Translation, pages 353-60, Lisboa, Portugal. Association for Computational Linguistics.

Ueffing, N. and Ney, H. 2005. Word-level Confidence Estimation for Machine Translation Using Phrased-based Translation Models. In Human Language Technology Conference and Conference on Empirical Methods in NLP, 763-70, Vancouver.

Ueffing, N. and Ney, H. 2007. Word-level confidence estimation for machine translation. Computational Linguistics, 33(1):9-40.

Ueffing, N., Macherey, K. and Ney, H. 2003. Confidence Measures for Statistical Machine Translation. In MT Summit IX, 394-401, New Orleans, LA.

Ueffing, N., Och, F.J. and Ney, H. 2002. Generation of Word Graphs in Statistical Machine Translation. In Conference on Empirical Methods for Natural Language Processing (EMNLP 02), 156 -63, Philadelphia, PA.

Watanabe, T., Suzuki., Tsukada, H. and Isozaki, H. 2007. Online large-margin training 
for statistical machine translation. In Proceedings of the 2007 Joint Conference on Empirical Methods in Natural Language Processing and Computational Natural Language Learning, 64-73, Prague, Czech Republic.

Wisniewski, G., Pécheux, N., Allauzen, A. and Yvon, F. 2014. Limsi submission for wmt'14 qe task. In Proceedings of the Ninth Workshop on Statistical Machine Translation, Baltimore, Maryland, USA.

Xiong, D., Zhang, M. and Li, H. 2010. Error Detection for Statistical Machine Translation Using Linguistic Features. In Proceedings of the 48th Annual Meeting of the Association for Computational Linguistics, 604-11, Uppsala, Sweden.

Zhang, Y., Almut, S.H. and Stephan, V. 2006. Distributed language modeling for n-best list re-ranking. In Proceedings of the 2006 Conference on Empirical Methods in Natural Language Processing (EMNLP 2006), 216-23, Sydney. 